\title{
Subcutaneous metastasis after three surgeries for brain metastasis from lung cancer: A case report and review of the literature
}

\author{
JI-QING QIU ${ }^{1 *}$, YU CUI $^{2 *}$, LI-CHAO SUN ${ }^{3}$ and ZHAN-PENG ZHU ${ }^{1}$ \\ Departments of ${ }^{1}$ Neurosurgery, ${ }^{2}$ Otolaryngology and ${ }^{3}$ Emergency, First Hospital \\ of Jilin University, Changchun, Jilin 130021, P.R. China
}

Received July 25, 2016; Accepted September 23, 2016

DOI: $10.3892 / \mathrm{mco} .2016 .1039$

\begin{abstract}
Lung cancer is a common malignancy that is frequently associated with distant subcutaneous metastasis. However, reports of subcutaneous metastasis of lung cancer after three surgeries for recurrent brain metastasis are scarce. The present study describes the case of a 49-year-old female patient who was admitted to our hospital with a cutaneous mass. The patient had a history of lung cancer and had undergone three surgeries for brain metastases. The subcutaneous mass was considered to be an implantation metastasis from the previous brain metastases. The cutaneous mass was grossly resected and histopathological examination revealed adenocarcinoma. This case highlights the need to perform a comprehensive analysis for suspected subcutaneous masses in lung cancer patients. Furthermore, pathological examination is crucial for accurate diagnosis and timely treatment.
\end{abstract}

\section{Introduction}

Lung cancer is one of the most common types of cancer worldwide, it is highly aggressive and has a high rate of distant metastasis (1). Subcutaneous metastasis from lung cancer has been well described (2); however, reports of subcutaneous metastasis of lung cancer after three surgeries for recurrent brain metastasis are scarce. We herein report a rare case of subcutaneous metastasis from pulmonary adenocarcinoma and provide a brief review of the relevant literature.

Correspondence to: Dr Zhan-Peng Zhu, Department of Neurosurgery, First Hospital of Jilin University, 71 Xinmin Street, Changchun, Jilin 130021, P.R. China

E-mail: 282324491@qq.com

${ }^{*}$ Contributed equally

Abbreviations: MRI, magnetic resonance imaging; CT, computed tomography; TTF-1, thyroid transcription factor-1; CK, cytokeratin

Key words: subcutaneous mass, implantation metastasis, lung cancer, surgery

\section{Case report}

A 49-year-old female patient was admitted to our hospital due to a fast-growing mass in the left temporal scalp at a craniotomy site (Fig. 1). The mass was round and adhered firmly to the scalp tissues. The patient was aware of the swelling as it caused her pain. A routine magnetic resonance imaging (MRI) revealed recurrent lesions in the left temporal and parietal lobes and a separate large nodular mass in the subcutaneous tissue coinciding with the site of the previous craniotomy (Fig. 2). In 2011, a lung computed tomography (CT) scan at another hospital revealed a $2.4-\mathrm{cm}$ mass in the upper lobe of the left lung. Fine-needle aspiration cytology revealed pulmonary adenocarcinoma. The patient did not undergo surgery due to enlarged mediastinal lymph nodes, but was treated with radiosurgery (5 years) and chemotherapy (2 years). The subsequent CT scan demonstrated no residual or recurrent adenocarcinoma in the lung.

In March, 2013, a routine MRI scan at another hospital revealed brain metastases in the left temporal and parietal lobes. The patient underwent subtotal resection of the tumors and decompressive craniectomy at the First Hospital of Jilin University (Changchun, China). In May, 2014 and August, 2015 the patient suffered recurrent brain metastases in the left temporal lobe, which were resected. The pathological diagnosis was adenocarcinoma.

In November, 2015, the patient was admitted to our hospital due to a fast-growing mass in the left temporal scalp at the craniotomy site.

The patient's medical history was unremarkable, apart from her father having been a heavy smoker for 30 years. The physical and neurological examinations were normal. There were no other metastases. The laboratory test results for tumor markers were as follows: Carcinoembryonic antigen, $191.6 \mathrm{ng} / \mathrm{ml}$ (normal, <3.4 ng/ml) and carbohydrate antigen 15-3, $38.03 \mathrm{U} / \mathrm{ml}$ (normal, <25.0 U/ml). Peripheral blood cell counts, liver and renal function tests and hormonal levels were within the normal range.

As the location of the mass corresponded to the site of the craniotomy, seeding was considered. Surgical resection of the subcutaneous nodule was performed. During surgery, the mass was found to be infiltrating the subcutaneous tissue and dura mater. There was no distinct boundary between the tumor and normal skin. The nodule contained numerous 
vessels (Fig. 3A). Total excision of the lesions in the left temporal and parietal lobes and the mass in the subcutaneous tissue was performed (Fig. 3B).

On immunohistochemistry (Fig. 4), the masses diffusely expressed thyroid transcription factor-1 (TTF-1) and cytokeratin (CK)7, the Ki-67 index was $25 \%$, whereas villin, CK20 and Wilms tumor-1 were negative. The patient continued her treatment and was followed up at another hospital.

\section{Discussion}

Cutaneous metastasis is caused by primary cancer-derived cells that grow in the skin (3). According to the published literature, the overall incidence of cutaneous metastasis is $2.9-5.3 \%$ (4), and $1-12 \%$ in lung cancer $(2,5-8)$. Cutaneous metastasis usually presents as solitary or multiple nodules sized $5 \mathrm{~mm}-10 \mathrm{~cm}$ that are firm, immobile and covered with normal skin. In the present case, the nodule was firm, relatively immobile, and of normal yellow color.

In the present case, the differential diagnosis was primary skin malignancy. When the primary tumor is an adenocarcinoma, other diagnoses such as melanoma and hematopoietic malignancies should be considered (9). The patient in the present study had undergone multiple surgeries with subsequent complications associated with the surgical wounds, which may have contributed to the scalp metastasis. Therefore, the initial diagnosis was subcutaneous metastasis of lung cancer. Certain cases of subcutaneous metastasis of lung cancer may manifest as purple or bright red masses that are ulcerated or cauliflower-like, accompanied by bleeding $(3,10)$. The patient in the present case did not experience bleeding, as the mass was discovered early.

The scalp accounts for 4-6.9\% of all cutaneous metastases and it is a relatively frequent metastatic site, possibly due to the abundant blood supply and immobility (4). Skin metastases from internal malignancies tend to occur at a site near the primary tumor $(11,12)$ through various routes, including lymphatic spread, hematogenous spread and direct implanting. The patient underwent three surgeries for brain metastasis and the mass was located at the surgical site. Therefore, the mass was considered to be an implantation metastasis from the previous brain metastases.

The treatment options for scalp metastasis include surgery, chemotherapy and radiotherapy. Our patient reported swelling and pain; therefore, she underwent surgical resection in an attempt to provide local palliation (13). During surgery, the mass was found to be infiltrating the subcutaneous tissue and dura mater. Surgical seeding of tumor cells is a known complication of lung cancer surgery (14). We considered that subcutaneous spreading of the intracranial tumor may have occurred, rather than metastasis from the lung. The localization of a metastasis along a surgical incision site indicates tumor seeding as the likely mechanism, particularly when a metastasis is apparent on imaging soon after surgery, as in this case. We consider that scalp metastases due to intraoperative seeding may occur in all histopathological grades of lung cancer.

The patient in the present case was at high risk for extracranial spreading of intracranial tumors, as she had undergone multiple surgeries. Surgical bone defects may compromise the natural

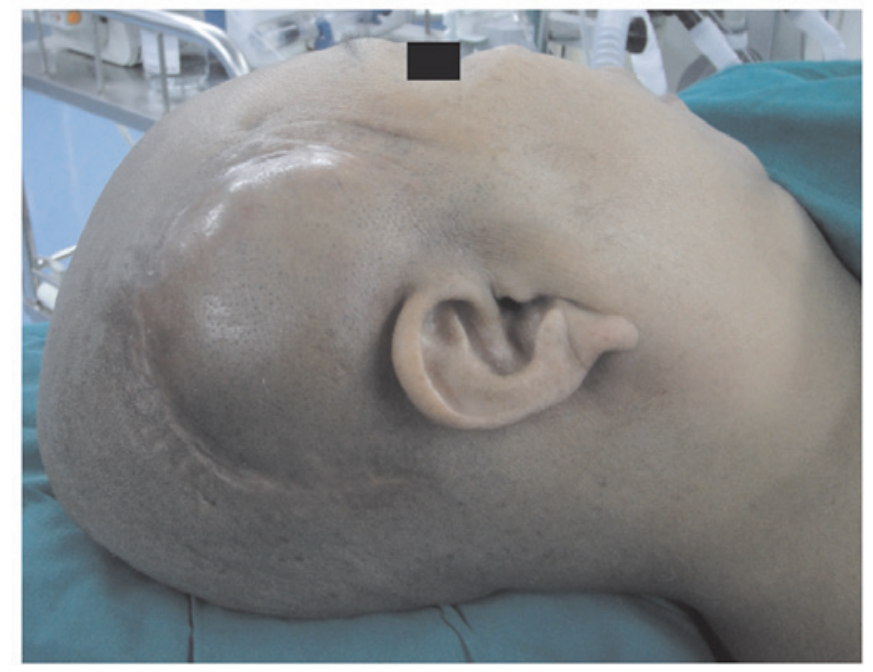

Figure 1. Preoperative photograph showing the subcutaneous mass.
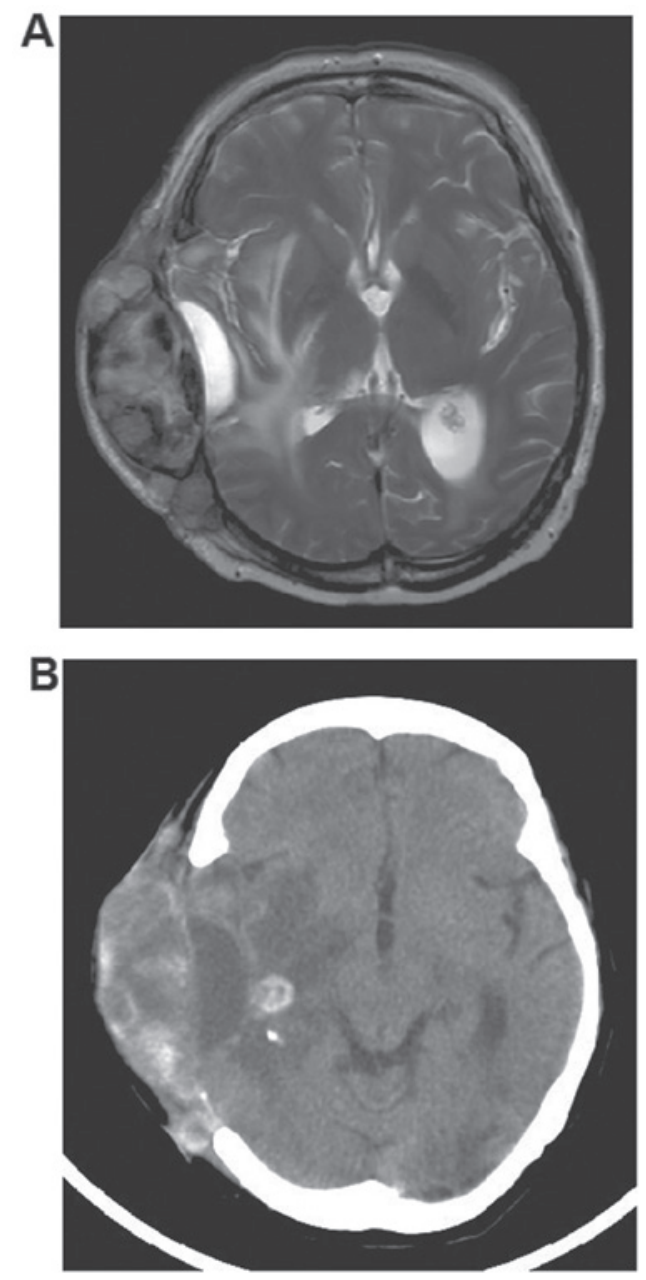

Figure 2. Preoperative neuroimaging: (A) Axial T2-weighted magnetic resonance imaging and (B) computed tomography scan revealed recurrent lesions in the left temporal and parietal lobes and a separate large nodular mass in the subcutaneous tissue coincident with the site of the previous craniotomy.

barriers to dissemination of intracranial tumors, providing access to the lymphatic system, blood vessels and connective tissues. Vascular invasion of the extracranial tissues, migration 

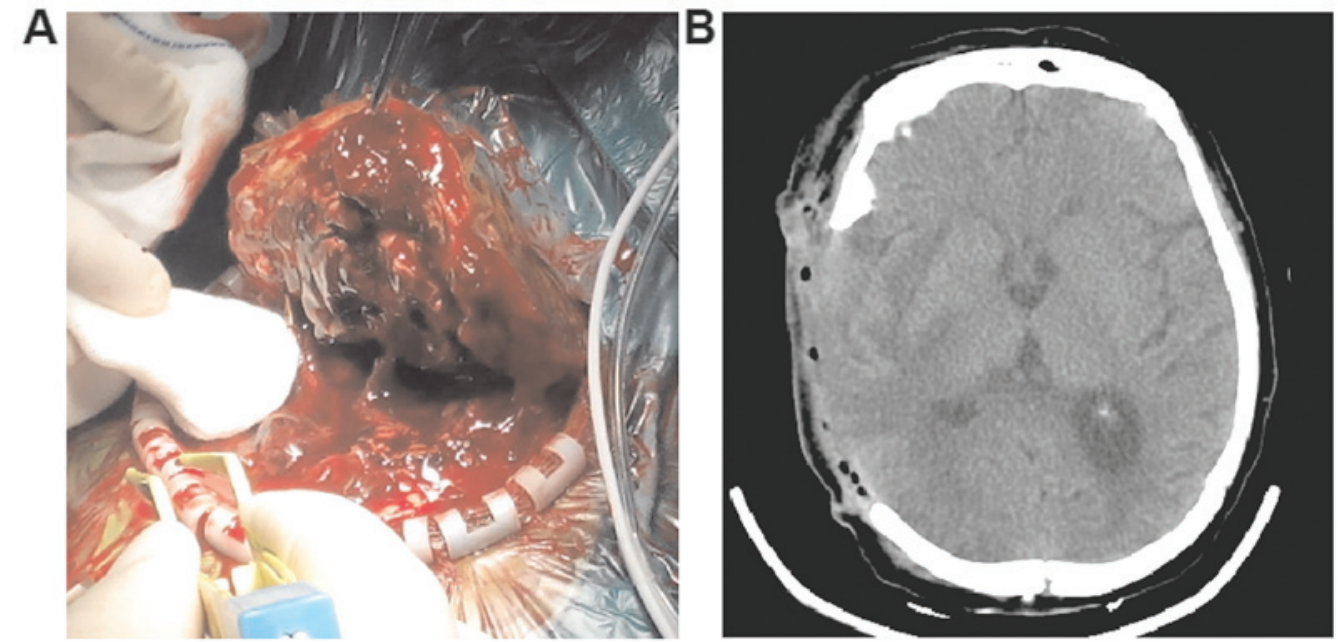

Figure 3. (A) Intraoperative imaging: The subcutaneous mass was highly vascular (B) Postoperative neuroimaging: Computed tomography scan showing the mass was completely resected.
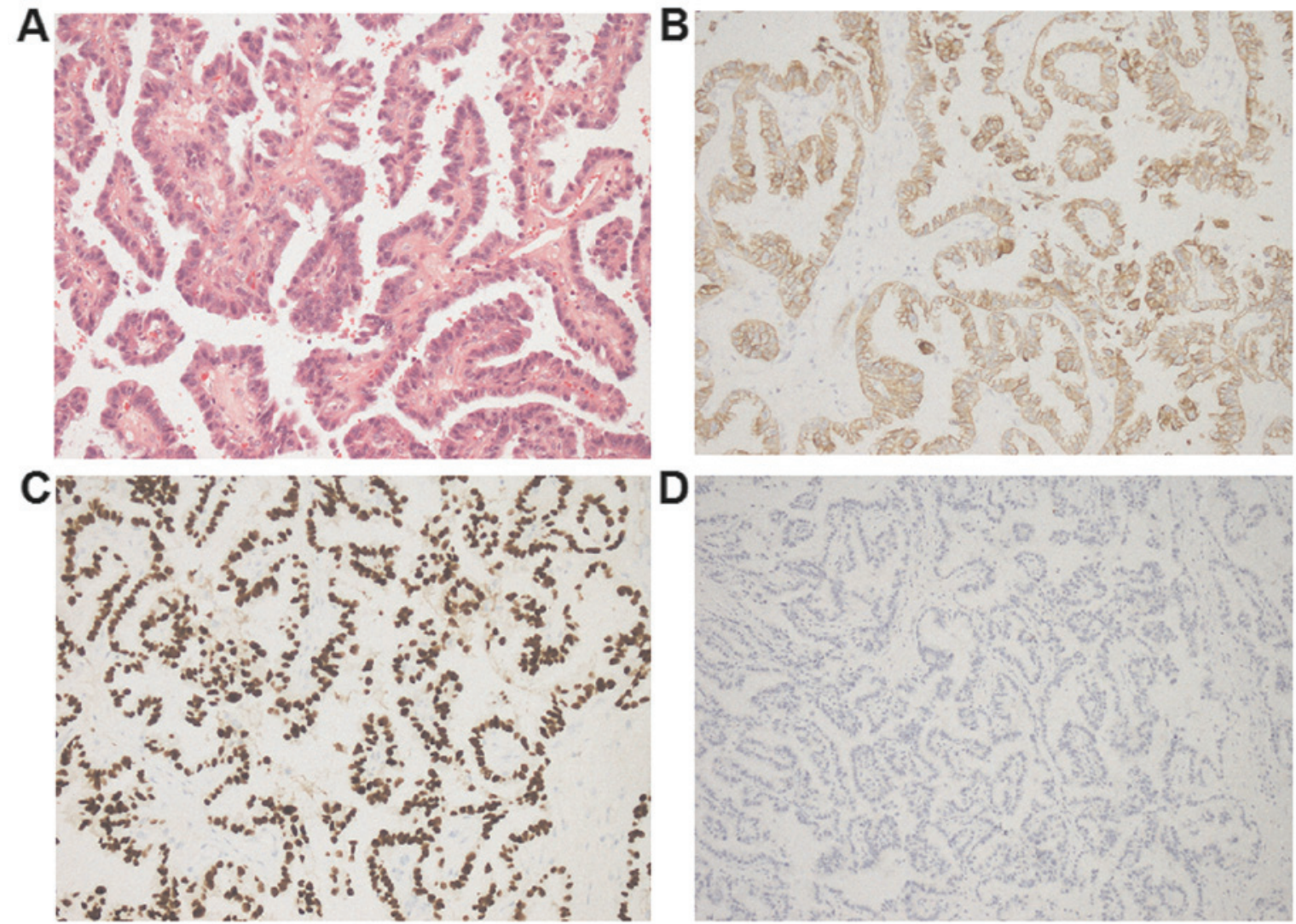

Figure 4. Immunohistochemistry confirmed the adenocarcinoma originated from the lung (A) Haematoxylin and eosin staining (magnification, x20); the tumor was (B) cytokeratin (CK)7-positive (magnification, x20) (C) thyroid transcription factor-1-positive (magnification, x20); and (D) CK20-negative (magnification, $\mathrm{x} 10)$.

of neoplastic cells and further growth may potentiate extracranial metastases. However, differentiation between implantation of tumor cells and metastasis from the lung may be difficult. Prevention of extracranial spreading of intracranial tumors following resection may be achieved by appropriate closure of the dura mater, changing surgical instruments and gloves for wound closure after the intracranial phase of the surgery, and copious saline irrigation of the wound prior to closure.
In our patient, diagnosis was made by histopathological examination of the cutaneous nodule following surgical excision. Histological study of the nodule revealed adenocarcinoma. The histology of cutaneous metastasis from lung cancer most commonly reveals adenocarcinoma, followed by squamous/small-cell carcinoma, and large-cell carcinoma (8). Immunohistochemistry, particularly CK7/CK20 (15) and TTF-1 is useful for identification of adenocarcinoma. In the 
present case, immunohistochemistry for CK7/CK20 was CK7-positive and for CK20-negative. TTF-1, a tissue-specific transcription factor expressed in epithelial cells of the thyroid gland and lung (16), confirmed that the primary origin of the adenocarcinoma was the lung.

Following three surgeries for brain metastases and one surgery for cutaneous metastasis, the patient recovered well and was able to care for herself. The presence of cutaneous metastases in lung cancer is associated with a poor prognosis, as this is an indication that the primary cancer is advanced. The median survival is $\sim 4$ months. The patient in the present case was followed up for $>4$ months and continues to show good recovery.

The present study was approved by the Ethics Committee of the First Hospital of Jilin University. Informed patient consent was obtained regarding the publication of the case details and accompanying images.

\section{Acknowledgements}

The present study has been edited and proofread by Medjaden Bioscience Limited.

\section{References}

1. Jemal A, Bray F, Center MM, Ferlay J, Ward E and Forman D: Global cancer statistics. CA Cancer J Clin 61: 69-90, 2011.

2. Salemis NS, Veloudis G, Spiliopoulos K, Nakos G, Vrizidis N and Gourgiotis S: Scalp metastasis as the first sign of small-cell lung cancer: Management and literature review. Int Surg 99: 325-329, 2014.

3. Riahi RR and Cohen PR: Clinical manifestations of cutaneous metastases: A review with special emphasis on cutaneous metastases mimicking keratoacanthoma. Am J Clin Dermatol 13: 103-112, 2012.

4. Krathen RA, Orengo IF and Rosen T: Cutaneous metastasis: A meta-analysis of data. South Med J 96: 164-167, 2003.
5. McGrath RB, Flood SP and Casey R: Cutaneous metastases in non-small cell lung cancer. BMJ Case Rep 2014: 2014. http://dx.doi.org/10.1136/bcr-2014-205752.

6. Perisano C, Spinelli MS, Graci C, Scaramuzzo L, Marzetti E, Barone C, Fabbriciani C and Maccauro G: Soft tissue metastases in lung cancer: A review of the literature. Eur Rev Med Pharmacol Sci 16: 1908-1914, 2012.

7. Kamble R, Kumar L, Kochupillai V, Sharma A, Sandhoo MS and Mohanti BK: Cutaneous metastases of lung cancer. Postgrad Med J 71: 741-743, 1995.

8. Song Z, Lin B, Shao L and Zhang Y: Cutaneous metastasis as a initial presentation in advanced non-small cell lung cancer and its poor survival prognosis. J Cancer Res Clin Oncol 138: 1613-1617, 2012.

9. Spitz DJ, Reddy V, Selvaggi SM, Kluskens L, Green L and Gattuso P: Fine-needle aspiration of scalp lesions. Diagn Cytopathol 23: 35-38, 2000.

10. Triller Vadnal K, Triller N, Pozek I, Kecelj P and Kosnik M: Skin metastases of lung cancer. Acta Dermatovenerol Alp Pannonica Adriat 17: 125-128, 2008.

11. Lookingbill DP, Spangler N and Helm KF: Cutaneous metastases in patients with metastatic carcinoma: A retrospective study of 4020 patients. J Am Acad Dermatol 29: 228-236, 1993.

12. Mollet TW, Garcia CA and Koester G: Skin metastases from lung cancer. Dermatol Online J 15: 1, 2009.

13. Saeed S, Keehn CA and Morgan MB: Cutaneous metastasis: A clinical, pathological, and immunohistochemical appraisal. J Cutan Pathol 31: 419-430, 2004.

14. Scotti V, Di Cataldo V, Falchini M, Meattini I, Livi L, Ugolini D, Comin CE, Mazza E, Franzese C and Biti G: Isolated chest wall implantation of non-small cell lung cancer after fine-needle aspiration: A case report and review of the literature. Tumori 98: $126 \mathrm{e}-129 \mathrm{e}, 2012$.

15. Rubin BP, Skarin AT, Pisick E, Rizk M and Salgia R: Use of cytokeratins 7 and 20 in determining the origin of metastatic carcinoma of unknown primary, with special emphasis on lung cancer. Eur J Cancer Prev 10: 77-82, 2001.

16. Azoulay S, Adem C, Pelletier FL, Barete S, Francès C and Capron F: Skin metastases from unknown origin: Role of immunohistochemistry in the evaluation of cutaneous metastases of carcinoma of unknown origin. J Cutan Pathol 32: 561-566, 2005. 